\title{
Susceptibilité magnétique du gadolinium pur en fonction du champ dans la région de réorientation de spins
}

\author{
A. BOUKRAA, P. VAJDA* et J.N. DAOU \\ "Hydrogène dans les Metaux", L.S.A.I., U.A. 775 du C.N.R.S., Bâtiment 350, Université Paris-Sud, \\ 91405 Orsay, France \\ "aussi au Laboratoire des Solides Irradies, Ecole Polytechnique, 91128 Palaiseau, France
}

Résumé.- La susceptibilité magnétique $\chi$ d'un polycristal de gadolinium, de pureté nominale $99,99 \%$, est mesurée en fonction de la température (110 $\mathrm{K} \leq \mathrm{T} \leq 295 \mathrm{~K})$ et du champ magnétique appliqué $(0,3 \mathrm{G} \leq \mathrm{H} \leq 2600 \mathrm{G})$ par la méthode du champ alternatif. Pour un champ $H$ faible et $T$ croissant, $\chi(T)$ passe par un maximum à $\mathrm{T}_{\mathrm{M}}=208 \mathrm{~K}$, diminue ensuite légèrement jusqu'à la température $T_{2}=223 \mathrm{~K}$ et chute rapidement au-delà. Avec l'augmentation de $\mathrm{H}$, la température correspondant à la chute brutale de $\chi$ diminue. La température $T_{M}$, par contre, n'est pas affectée par $H$ tant que celui-ci n'a pas dépassé un champ critique $H_{c}$. Au-dessus de $H_{c}$, l'allure de $\chi(T)$ change complètement et à $H=1040 \mathrm{G}$, le maximum $T_{M}$ se situe à $141 \mathrm{~K}$ et $T_{2}$ correspond à un minimum local de $\chi$. Nous discuterons ces effets en fonction de l'angle variable que présentent les spins ferromagnétiques avec l'axe $c$ de la structure hexagonale de Gd. Nous noterons enfin que dans un polycristal de pureté $99,9 \%$ une telle structure fine de $\chi$ autour de $T_{2}$ n'est plus constatée.

\begin{abstract}
A.c. magnetic susceptibilities $\chi$ of a $99.99 \%$ nominal purity gadolinium polycrystal have been measured in the temperature range 110 - $295 \mathrm{~K}$ as a function of applied magnetic fields $(0.3 \mathrm{G} \leq \mathrm{H} \leq 2600 \mathrm{G})$. For weak fields and increasing temperature, $\chi(T)$ goes through a maximum at $T_{M}=208 \mathrm{~K}$ then it decreases slightly until it reaches the temperature $T_{2}=$ $223 \mathrm{~K}$ where it falls precipitously. The temperature corresponding to the sharp fall of $\chi$ decreases when $H$ is augmented but $T_{M}$ remains unaffected as long as $\mathrm{H}$ has not reached a critical field $H_{c}$. The shape of $\chi(T)$ changes drastically above $H_{c}$; for $H=1040 \mathrm{G}$ for example $T_{M}$ shifts to $141 \mathrm{~K}$ and $T_{2}$ emerges as a local minimum in $\chi$. We discuss these effects in terms of the variable angle between the ferromagnetic spins and the hexagonal c-axis of $\mathrm{Gd}$. This fine structure of $\chi$ in the region of $\mathrm{T}_{2}$ has not been detected in a 99.9\% pure polycrystal.
\end{abstract}




\section{Introduction.}

A la température de Curie $(\approx 293 \mathrm{~K})$, les moments magnéliques du gadolinium métallique s'ordonnent ferromagnétiquement en s'alignant en moyenne avec l'axe $c$ de la structure hexagonale. Au-dessous de $\mathrm{T}_{\mathrm{r}} \approx 235 \mathrm{~K}$, appelée température de réorientation de spins, les moments s'écartent brutalement de l'axe $c$ d'un angle $\theta$ dont la dépendance en température a été précisée par Cable et al [1], $\theta$ atteignant un maximum d'environ $72^{\circ}$ à $\mathrm{T} \approx 224 \mathrm{~K}$. Les moments décrivent alors, pour un domaine ferromagnétique donné, un cône de directions d'aimantation facile autour de l'axe c. L'intervalle de températures 224-235 K coïncide avec de nombreuses singularités observées dans les propriétés du gadolinium [2-6]. La constante d'anisotropie magnétocristalline $K_{1}$, qui est donnée par l'énergie d'anisotropie magnétique $E_{k} \approx K_{1} \sin ^{2} \theta+K_{2} \sin ^{4} \theta\left(K_{2}>0\right)$ [7], devient négative pour $T<T_{r}$ et serait ainsi responsable de cette transition de phase du second ordre. Plusieurs auteurs trouvent un point critique près de $210 \mathrm{~K}$ pour certaines propriétés magnétiques [8-13]. De plus, Milstein et al [12] observent une structure supplémentaire dans la partie réelle de la perméabilité près de $230 \mathrm{~K}$ et Ponomarev [14] détermine un pic à 224-229 $\mathrm{K}$ dans la partie imaginaire.

Dans cette communication, nous présentons des mesures de la susceptibilité magnétique $\chi$ d'un échantillon de gadolinium pur en fonction d'un champ magnétique appliqué en concentrant notre attention sur le domaine autour de $T_{r}$.

\section{Méthode Expérimentale.}

Deux échantillons polycristallins de gadolinium de pureté nominale $99,99 \%$ $(4 \mathrm{~N})$ et $99,9 \%(3 \mathrm{~N})$ et de dimensions respectives $1,1 \times 1,8 \times 5,0 \mathrm{~mm}^{3}$ et $1,9 \times 2,4 \times 5,2$ $\mathrm{m} \mathrm{m}^{3}$ sont utilisés. Les principales impuretés métalliques dans l'échantillon $4 \mathrm{~N}$ (brut de préparation) fourni par Ames Laboratory, Iowa, sont en p.p.m. atomique: $67 \mathrm{Fe}, 20 \mathrm{Al}, 15 \mathrm{Ni}, 9 \mathrm{Th}, 7 \mathrm{Si}, 5 \mathrm{Cr}, 5 \mathrm{Y}$ tandis que la teneur en oxygène y est de 255 p.p.m. atomique.

La partie réelle (composante en phase) de la susceptibilité magnétique $\chi$ est mesurée par la méthode du champ alternatif (fréquence $=533 \mathrm{~Hz}$, champ alternatif efficace $\leq 1 \mathrm{G}$ ) dans un cryostat à azote liquide entre $110 \mathrm{~K}$ et $295 \mathrm{~K}$ en fonction d'un champ magnétique statique déterminé à $\pm 2 \%$. L'erreur absolue sur $\chi$ est estimée à $\pm 10 \%$ alors que, pour une remontée en température, son erreur relative est inférieure ou égale à $0,3 \%$. La température est déterminée à $\pm 0,5 \mathrm{~K}$ par une résistance étalonnée de platine. Seul le spécimen le plus pur est étudié en fonction du champ, celui-ci n'étant appliqué que lors de la mesure c'est-à-dire en remontée de température (à partir de $80 \mathrm{~K}$ ). Dans tous les cas, l'axe de la bobine de détection et l'orientation du champ statique, si celui-ci est appliqué, sont parallèles à la plus grande dimension d'une plaquette. Nous portons sur les figures la susceptibilité apparente $\chi_{\text {app }}$ qui n'a pas été corrigée pour le facteur démagnétisant, essentiellement indépendant de la température, $N\left(\chi_{\text {app }}{ }^{-1}=\chi^{-1}+N\right)$.

\section{Résultats et Discussion.}

La susceptibilité magnétique $\chi$ du gadolinium $4 \mathrm{~N}$ dans un champ faible $(H \approx$ $0,3 \mathrm{G})$ augmente trés légèrement entre $110 \mathrm{~K}$ et $208 \mathrm{~K}$, atteignant un maximum à $\mathrm{T}_{\mathrm{M}}$ $=208 \mathrm{~K}$ et diminuant ensuite faiblement; à $\mathrm{T}_{2}=223 \mathrm{~K}$, elle subit une chute brutale qui s'accentue juste avant la température de Curie $T_{\mathfrak{c}}$ (pic à 292,8 $\mathrm{K}$ précédé par un minimum à $290,4 \mathrm{~K}$ )(figures 1 à 3 ). Nous interprétons cette chute rapide comme le début du retour des moments magnétiques de la direction d'angle $\theta$ maximum vers la direction de l'axe hexagonal en accord avec la variation de $\theta$ dans [1]. L'allure de $\chi(\mathrm{T})$ s'accorde avec le large maximum observé par Jelinek et al [10] à $215 \mathrm{~K}$.

L'échantillon $3 \mathrm{~N}$ présente un comportement analogue au $4 \mathrm{~N}$ à l'exception de l'amplitude de $\chi$ qui est plus faible et de la transition à $223 \mathrm{~K}$ qui n'est plus discernable (figures 1 et 3 ). La diminution d'amplitude peut être attribuée à un facteur démagnétisant différent mais il n'est pas exclu que la présence d'impuretés (notamment des inclusions d'oxides) et de défauts structurels atténue également $\chi[12,15]$. La disparition de la transition à $223 \mathrm{~K}$ est aussi le signe d'une texture écrouie car plusieurs auteurs rapportent l'absence de singularité à $T_{r}$ dans 
des échantillons non-recuits $[2,4,12,16]$.

Avec l'application d'un champ magnétique (à la température de l'azole liquide) au spécimen $4 \mathrm{~N}$, on distinguera trois régions:

a) $\mathrm{H} \leq 500 \mathrm{G}$. Le champ a pour effet de diminuer la température $\mathrm{T}_{2}$ de la chute brutale de $\chi$ (figures 3 et 4), impliquant que la direction d'aimantation facile commence à revenir vers la direction de l'axe hexagonal $c$ à des températures plus basses. Cette diminution est effective même pour $H$ trés faible. Le champ, par contre, n'affecte ni la position du maximum à $\mathrm{T}_{M}=208 \mathrm{~K}$ ni la valeur absolue de $\chi$ au-dessous de $T_{M}$.

b) $500 \mathrm{G} \leq \mathrm{H} \leq 1100 \mathrm{G}$. En augmentant $\mathrm{H}$, l'amplitude de $\chi$ et la température du maximum $\mathrm{T}_{\mathrm{M}}$ diminuent et, à partir de $\mathrm{H}=417 \mathrm{G}$, un coude, auquel sont associés deux points d'inflexion, apparaît dans la courbe $\chi(\mathrm{T})$ (figures 1 et 2). A tous les champs appliqués, l'un des points d'inflexion reste fixé autour de $224 \mathrm{~K}$. L'autre, qu'on désignera par $T_{1}$, diminue comme $T_{M}$ avec l'augmentation du champ (figure 4). $\mathrm{A} H=1040 \mathrm{G}, \mathrm{T}_{\mathrm{M}}=141 \mathrm{~K}$, le point d'inflexion à $224 \mathrm{~K}$ devient aussi un minimum local et $\chi(T)$ décrit pratiquement un plateau dans l'intervalle 224-242 K.

c) $\mathrm{H}>1100 \mathrm{G}$. Pour les deux champs appliqués dans cette région (1521 et 2590 $\mathrm{G}), \chi$ diminue hyperboliquement avec la croissance de $\mathrm{T}$; cette diminution est régulière, sans aucune structure particulière.

Nous avons également mesuré $\chi$ en fonction du champ à une température fixe, $T=(83,7 \pm 1) \quad K$ (figure 4). Il est clair que l'aimantation ne commence à être saturée qu'à partir d'un champ plus grand que $600 \mathrm{G}$, la variation $\mathrm{d} \chi / \mathrm{dH}$ la plus rapide se produisant à $H_{c}=850 \mathrm{G}$ qu'on définira comme un champ critique. Audelà de ce champ, $\chi(H)$ suit une loi hyperbolique $(\chi=M / H=$ constante/H). La valeur de $H_{\mathcal{C}}$ est aussi en accord avec la variation de $\chi$ dans le domaine de champs 500-1100 G (figure 1).

Une analyse radiocristallographique sur un spécimen $4 \mathrm{~N}$ de mêrne provenance montre que le paramètre de réseau, $c$, possède une variation thermique conforme aux mesures de Darnell [17] avec une structure fine décelable dans la région de $T_{r}$. Cette structure fine, contenant plus d'une singularité, se mánifeste aussi dans la dépendance thermique du champ magnétique local au site d'un muon positif observée par Graf et al [16]. Le champ local accuse en effet une rapide diminution entre $210 \mathrm{~K}$ et $225 \mathrm{~K}$ environ, températures voisines respectivement de $T_{M}$ et $T_{2}$. Etant une sonde microscopique, le muon devrait généralement rester insensible aux imperfections de l'échantillon.

Ce travail a donc permis de mettre en évidence une structure fine dans la susceptibilité magnétique du gadolinium et a montré que l'état de pureté de ce métal a une grande influence sur l'observation de propriétés magnétiques macroscopiques autour de $T_{r}$.

\section{Réf ér ences}

*aussi au Laboratoire des Solides Irradiés, Ecole Polytechnique, 91128 Palaiseau, France

[1] Cable J.W., and Koehler W.C., J. Appl. Phys. 53 (1982) 1904-6

[2] Bodryakov V.Yu., Nikitin S.A., and Tishin A.M., Sov. Phys. Solid State 33 (1991)

$1260-2$

[3] Klimker H., and Rosen M., Phys. Rev. B 7 (1973) 2054-61

[4] Salamon M.B., and Simons D.S., Phys. Rev. B 7 (1973) 229-32

[5] Saleh A.G.A.M., and Saunders N.H., J. Magn. Magn. Mater. 29 (1982) 197-202

[6] Sousa J.B., Amado M.M., Pinto R.P., Pinheiro M.F., Moreira J.M., Braga M.E., Ausloos M., Clippe P., Hukin D., Garton G., and Walker P., J. Magn. Magn. Mater. 15 18 (1980) 892-4

[7] Belov K.P., Zvezdin A.K., Kadomtseva A.M., and Levitin R.Z., Sov. Phys. Usp. 19 (1976) $574-96$

[8] Belov K.P., Levitin R.Z., Nikitin S.A., and Ped'ko A.V., Sov. Phys. JETP 13 (1961) 1096-101

[9] Belov K.P., and Ped'ko A.V., Sov. Phys. JETP 15 (1962) 62-4

[10] Jelinek F.J., Hill E.D., and Gerstein B.C., J. Phys. Chem. Solids 26 (1965) 1475-88

[11] Milstein F., and Robinson L.B., Phys. Rev. 177 (1969) 904-9 
[12] Milstein F., and Zyvoloski G., J. Appl. Phys. 43 (1972) 4217-25

[13] Telesnin R.V., Al'meneva D.B., and Pogozhev V.A., Sov. Phys. Solid State 4 (1962) 256-8

[14] Ponomarev P.K., Phys. Stat. Sol. (a) 74 (1982) K165-8

[15] Smith R.L., Tanner B.K., and Corner W.D., J. Phys. F: Metal Phys. 7 (1977) L.22932

[16] Graf H., Kündig W., Meier P.F., and Patterson B.D., J. Appl. Phys. 49 (1978) 1549-51

[17] Darnell F.J., Phys. Rev. 130 (1963) 1825-8 


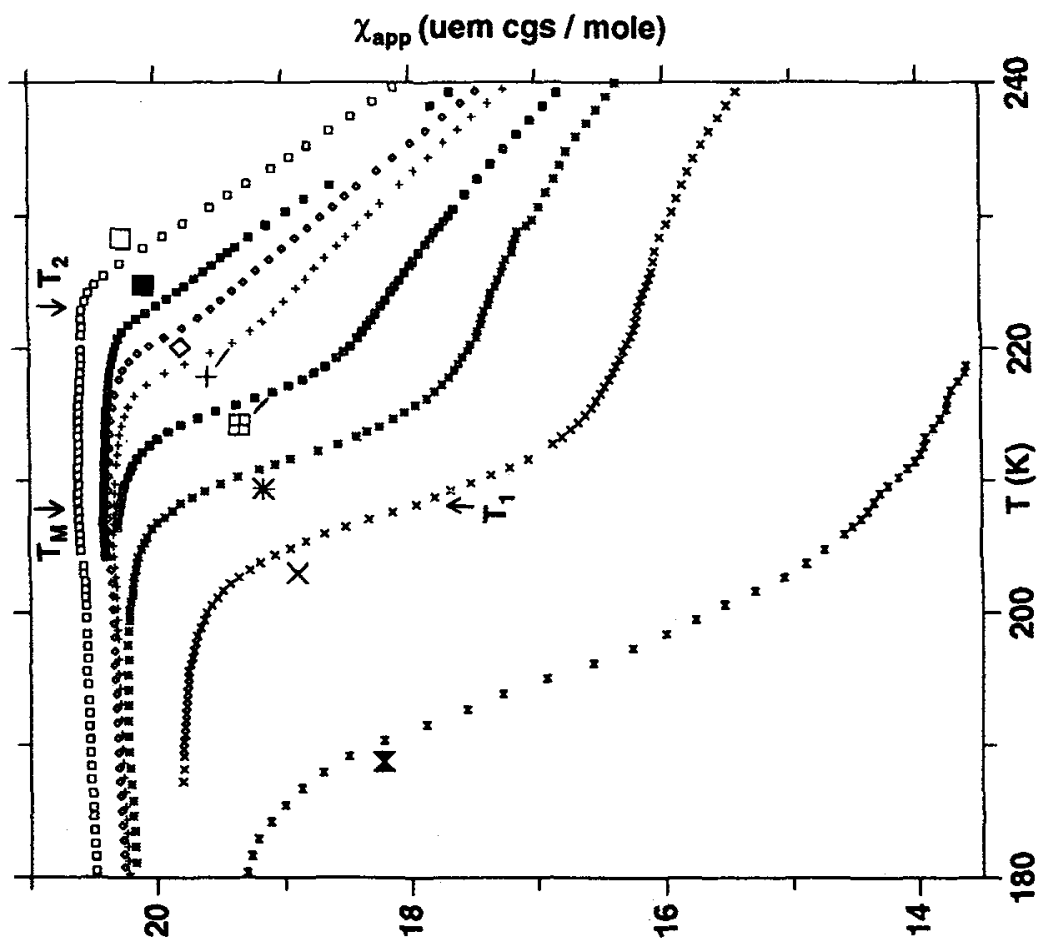

ธ붛ํ웜

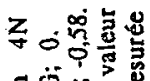

ह

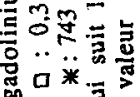

舟口承

z

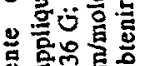

बें है

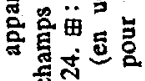

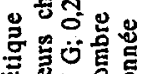

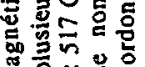

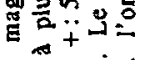

过完車

氙诺高

影

总落范紫

1 Уิ

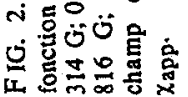

$\chi_{\text {app }}$ (uem cgs / mole)

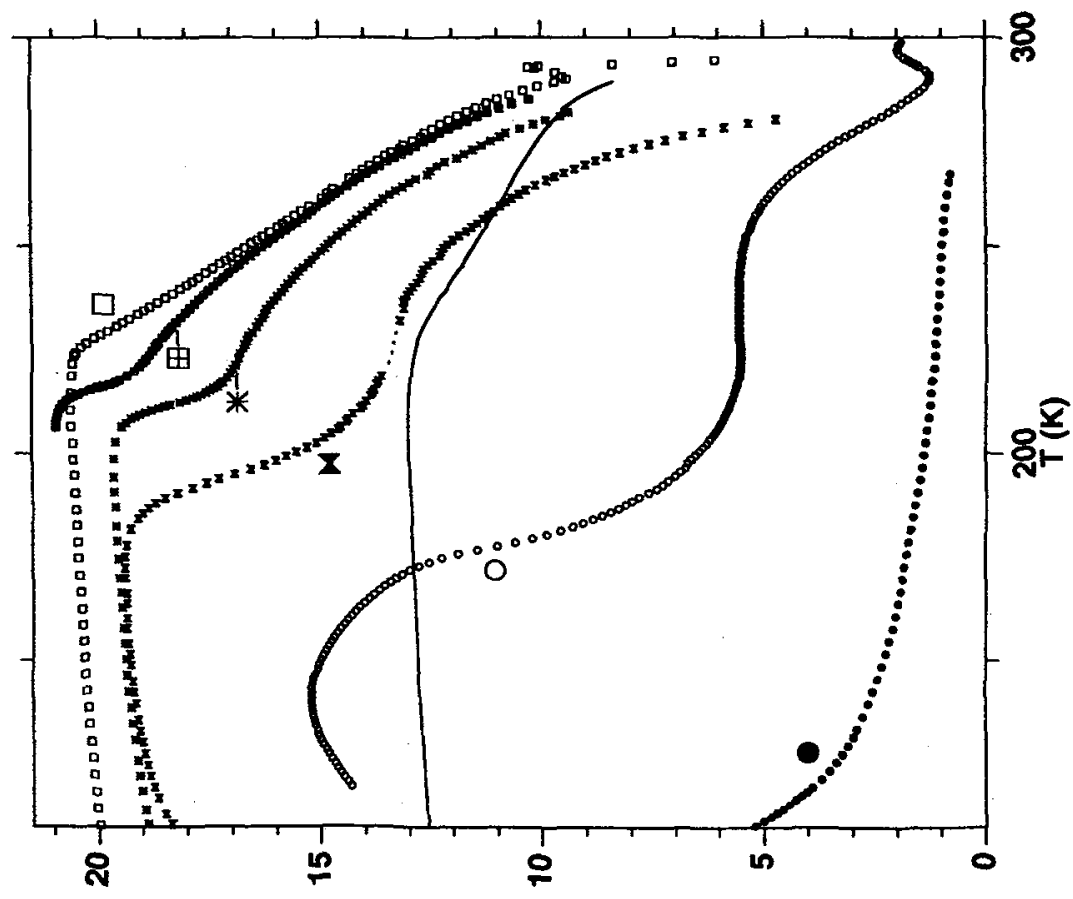

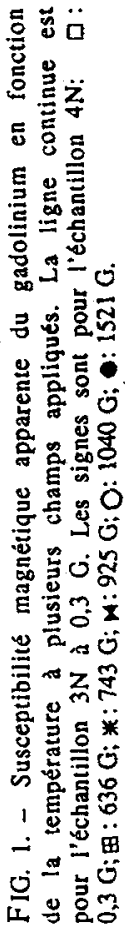




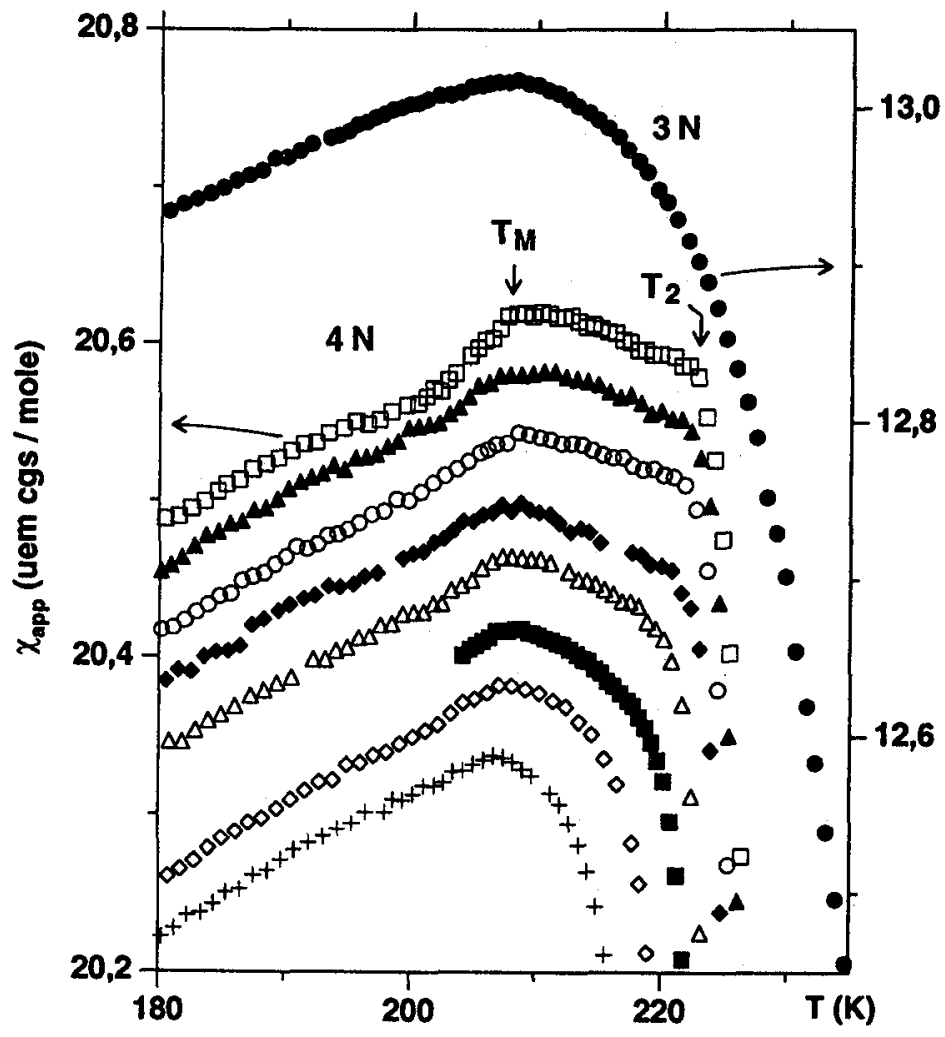

FIG. 3. - Susceptibilité magnétique apparente du gadolinium en fonction de la température à plusieurs champs appliqués. $\bullet$ : Echantillon $3 \mathrm{~N}$ à 0,3 G. Echantillon $4 \mathrm{~N}:$ 口: $0,3 \mathrm{G} ; 0 . \Delta: 20 \mathrm{G} ;-0.01$. O: $51 \mathrm{G}: 0,05$. $\$: 101 \mathrm{G}: 0.16 . \Delta:$ 218 G: 0.04. —: 314 G; 0,62 . $0: 417$ G; 0,64 . +: $517 \mathrm{G} ; 0,24$. Le nombre (en uem/mole) qui suit la valeur du champ doit êre ajouté l'ordonné de gauche pour obtenir la valeur mesuré de $\chi_{\text {app. }}$.

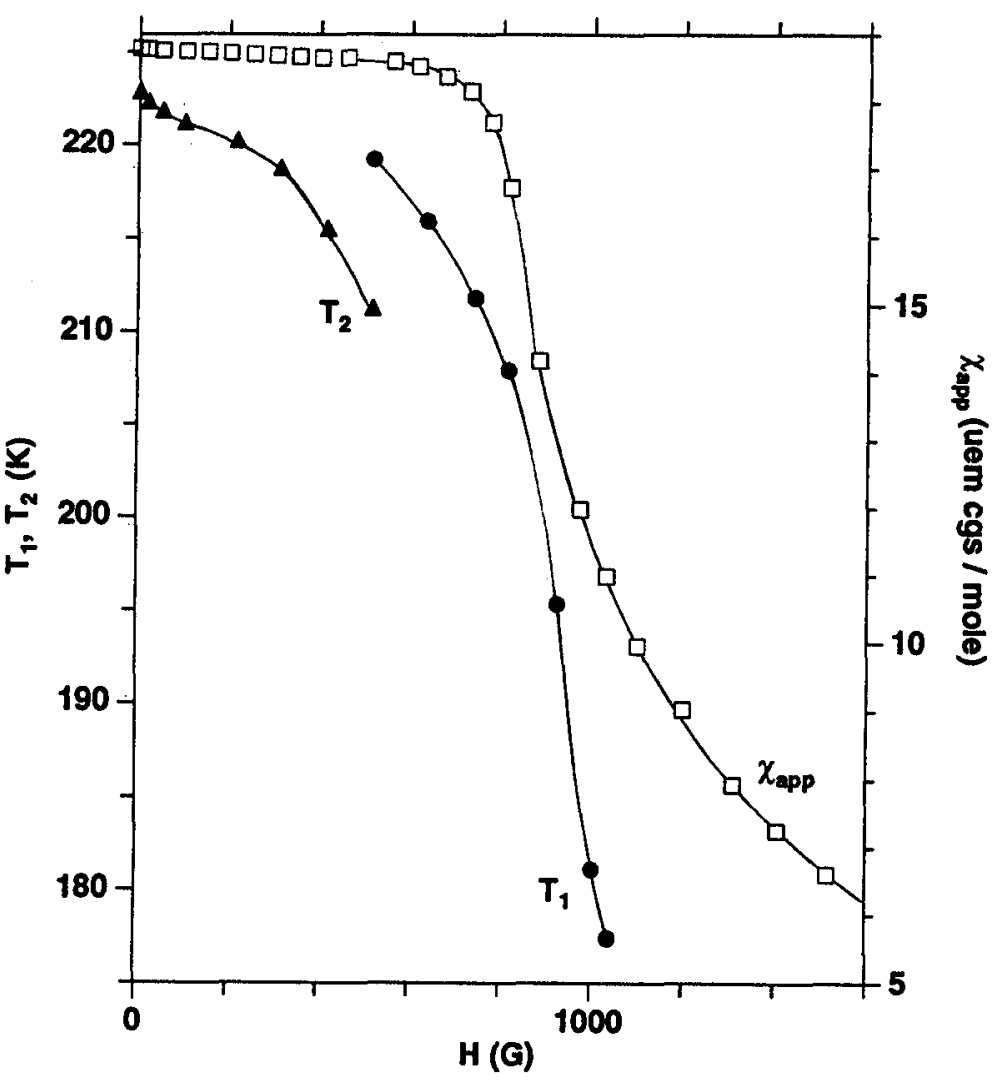

号

FIG. 4. - Ordonnee de gauche: Temperatures du point d'inflexion $T_{1}(\bullet)$ et de la chute rapide de $\chi, T_{2}(\Delta)$, en fonction du champ applique $H$. Ordonnte de droite: susceptibilité magnétique apparente $(\square)$ de l'échantillon $4 \mathrm{~N}$ aे $\mathrm{T}=(83,7 \pm 1) \mathrm{K}$ en fonction du champ applique $\mathrm{H}$. 